or in combination with other cellular defects and mutations ${ }^{20}$.

The work described here indicates that the centrosome in mammalian cells might have an essential function in cytokinesis and progression from G1 into $S$ phase. It is unclear whether centrosomes mediate these events directly, or whether centrosome defects trigger checkpoints that monitor cell-cycle progression. A better understanding of the mechanisms by which centrosomes influence these events will come from an appreciation of the molecular details of the pathways involved. One approach to this problem will be to identify and analyse proteins that are homologous to those involved in similar pathways in yeast. Another is to investigate further the centrosome components that have already been identified in metazoans. It has been proposed that some of the integral centriole/centrosome proteins that have been identified as autoantibody targets in autoimmune diseases might function in cell-cycle progression (ref. 21; S. Doxsey and R. Balczon, unpublished observations). Further regulatory pathways might also be influenced by centrosomes, and the centrosomal localization of regulatory molecules that communicate with signal-transduction pathways indicates a possible connection between centrosomes and the extracellular environment. Elucidation of the links between centrosomes and these and other cellular pathways is likely to be a fertile area of future discovery.

Note added in proof: Additional references are refs 22 and 23.

Stephen J. Doxsey is in the Program in Molecular Medicine, University of Massachusetts Medical

School, 373 Plantation Street, Worcester,

Massachusetts 01605, USA.

e-mail:stephen.doxsey@umassmed.edu

1. Zheng, Y., Wong, M. L., Alberts, B. \& Mitchison, T. Nature 378, 578-583 (1995).

2. Moritz, M., Braunfield, M. B., Sedat, J. W., Alberts, B. \& Agard, D. A. Nature 378, 638-640 (1995).

3. Zimmerman, W., Sparks, C. A., \& Doxsey, S. J. Curr. Opin. Cell Biol. 11, 122-128 (1999).

4. Diviani, D. \& Scott, J. D. J. Cell Sci. 114, 1431-1437 (2001).

\title{
G-protein activation live
}

G proteins are commonly used in signal transduction pathways in animal cells in response to diverse stimuli such as light, odorants, hormones, neurotransmitters and chemoattractants. They consist of heterotrimers of an $\alpha$-subunit, a $\beta$-subunit and a $\gamma$ subunit. Upon ligand binding, G-protein-coupled receptors (GPCRs) catalyse the exchange of GDP to GTP on the $\alpha$-subunit. The GTP-bound $\alpha$-subunit is then thought to dissociate from the $\beta \gamma$-heterodimer, allowing each party to signal to its downstream effector. The activation is terminated by GTP hydrolysis by the GTPase domain of the $\alpha$-subunit, leaving the $\alpha$-subunit in the GDP-bound form and allowing heterotrimerization to resume.

Activation of heterotrimeric $\mathrm{G}$ proteins has so far been difficult to monitor directly in vivo in real time because of technical difficulties. Janetopoulos and colleagues now use fluorescence resonance energy transfer (FRET) technology to study the cycle of activation of heterotrimeric $\mathrm{G}$ proteins in time and space in vivo, shedding light on the kinetics and subcellular localization of heterotrimeric G proteins (Science 291, 2408-2411; 2001).

$\mathrm{G}$ proteins in the social amoeba Dictyostelium discoideum are activated by the binding of the chemoattractant cAMP to its receptor $\mathrm{CAR} 1$. This results in the recruitment and activation of effectors of polarized actin polymerization, membrane protrusion at the leading edge of the cell and cell migration towards the chemoattractant. Janetopoulos and colleagues expressed fusion constructs of the $\mathrm{G} \alpha_{2}$ subunit and cyan fluorescent protein (CFP) and of the G $\beta$ subunit and yellow fluorescent protein (YFP) in a $\mathrm{G}_{2}$ - or $\mathrm{G} \beta$-null background. These fusion proteins were shown to be fully functional in terms of signalling. On exposure to a light with a wavelength of $440 \mathrm{~nm}$, which excites the CFP fluorophore, energy is transferred from CFP to YFP when the two probes are in close proximity, and a fluorescent signal is emitted by the yellow fluorophore. When the fluorophores are too far apart, the energy cannot be transferred from CFP to YFP and there is no fluorescence emission from the YFP probe.

The interaction between the $\alpha$ - and $\beta$-subunits was thus monitored by exposing cells to a wavelength of $440 \mathrm{~nm}$ and recording a FRET fluorescence signal between 460 and $600 \mathrm{~nm}$. A FRET fluorescence emission signal was detected at $527 \mathrm{~nm}$ specifically in cells expressing both fusion constructs. Activation by cAMP results in a sharp decrease in FRET fluorescence, which suggests complete dissociation of the heterotrimer rather than just a change in conformation. The kinetics of activation is

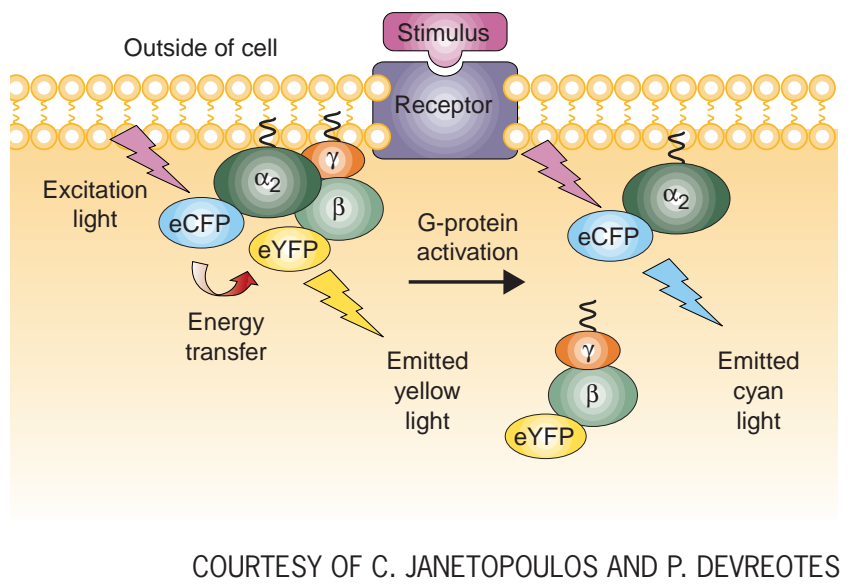

extremely rapid: maximum stimulation was reached less than 10 seconds after the addition of cAMP, and $90 \%$ of maximum activation was achieved in a matter of a few seconds. Activation is reversible, as the removal of cAMP resulted in a return to maximum fluorescence levels within 2 minutes. It is also dosedependent: the $\mathrm{EC}_{50}$ value for cAMP was estimated to be $10 \mathrm{nM}$. The $k_{\mathrm{d}}$ for binding of cAMP to cAMP receptor- 1 was approximately $180 \mathrm{nM}$, suggesting that the steady-state level of G-protein activation saturates before all receptors are occupied.

Sustained activation of GCPRs is known to result in adaptation, for example, of actin polymerization and other responses. As Janetopoulos and colleagues found that sustained exposure to cAMP leads to a prolonged loss of the FRET fluorescence signal, the mechanism of adaptation does not seem to be at the level of $\mathrm{G}$ proteins; it must be farther downstream.

Taken together, these observations also suggest that occupied receptors repeatedly activate $\mathrm{G}$ proteins, that regulators of Gprotein signalling (RGS) are likely to modulate the ratio of active to inactive $G$ proteins rather than the time course of activation, and that the distribution of activated $G$ proteins probably reflects the shallow gradient of receptor occupancy rather than the sharply localized physiological response at the leading edge of the cell. 